Stiftung Innovative Zahnmedizin

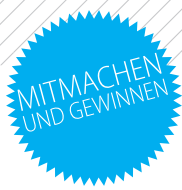

\title{
Gesamtdotierung EUR 10.000 - Innovationspreis 2010
}

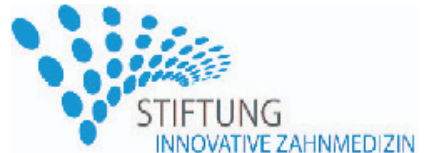

Der Dental Innovation Award, gestiftet von der Stiftung Innovative Zahnmedizin wird erstmalig im Jahr 2010 für herausragende innovative Behandlungen und Praxiskonzepte vergeben. Die Gesamtdotierung beträgt $€ 10.000$.

Preis für die Falldarstellung

Die moderne Kariesbehandlung orientiert sich an minimal-intervenierenden Grundsätzen.

Dies bedeutet neben der substanz- schonenden, defektorientierten invasiven Behandlung, eine am Kariesrisiko des jeweiligen Patienten ausgerichtete primär- und sekundärpräventive Betreuung. Der Preis prämiert die Darstellungen von Behandlungsfällen, die sich an diesen Grundsätzen orientieren. Hierbei fließen die Berücksichtigung der Anamnese, die Vorgehensweise bei Detektion-, Beurteilung und Diagnose von Zahnhartsubstanzdefekten unter Berücksichtigung von individuellen Faktoren, die Dokumentation der erhobenen Befunde, die Behandlungsplanung sowie die Durchführung der Therapie in die Beurteilung der Jury ein.

An dieser Ausschreibung können angehende und approbierte Ärzte und Zahnärzte aus Praxis, Universität und öffentlichem Gesundheitswesen teilnehmen.

Preis für innovatives Praxiskonzept

Dieser Preis prämiert innovative Praxiskonzepte, die sich an minimal-intervenierenden, wissenschaftlich basierten Grundsätzen orientieren.
Hierbei werden neben der ggf. an Patientenbeispielen dargestellten Anwendung aktueller di agnostischer und therapeutischer Hilfsmittel, die dargestellte Struktur, Organisation und Dokumentationsmöglichkeiten der Praxis zur Beurteilung des Innovationsgrades herangezogen.

An dieser Ausschreibung können approbierte Zahnärzte aus der Praxis teilnehmen. Einsendeschluss 31.12.2010

入 Tel. 040 - 63945223 www.stiftung-izm.com

\section{GlaxoSmithKline}

\section{X-trem gründlich bis in den Zahnzwischenraum}

\begin{abstract}
„Die Zähne sind sauber, wenn man beim Putzen mit der Zahnbürste kräftig drückt und der Arm weh tut", beschreibt der typische Schrubber seinen Putzerfolg. Der Techniker legt dagegen Wert auf ein Zahnputzinstrument, das ihm durch das Bürstendesign eine echte Unterstützung bei der täglichen Mundhygiene bietet. Besonders wichtig ist ihm die Erreichbarkeit der schwer zugänglichen Interdentalräume. Die neue Dr.BEST ${ }^{\circledR} X$ Zwischenzahn Zahnbürste hat schräg ange-winkelte Borsten, die mit ihrer Kreuz-Anordnung ideal entlang der Zahnflanken gleiten. Darüber hinaus gelangen die verlängerten X-Seidenfein-Borsten
\end{abstract}

40 Prozent tiefer in die engen Zahnzwischenräume im Vergleich zu herkömmlichen X-Borsten Zahnbürsten, wie eine Laborstudie aus den USA zeigt. „Die X-Seidenfein-Borsten der neuen Dr.BEST ${ }^{\circledR}$ Zahnbürste erreichen die Zwischenzahnflächen sechsmal besser als herkömmliche X-Borsten, fasst Dr. Miriam Dörfler von der Dr.BEST ${ }^{\oplus}$ Forschung die Ergebnisse der Tests zusammen.

In einer Verbraucherbewertung heben 96 Prozent der Anwender die insgesamt sehr gute Reinigungsleistung hervor. Darüber hinaus bestätigen 72 Prozent der Befragten der Dr.BEST ${ }^{\circledR}$ X-Zwischenzahn Zahnbürste eine Reinigung der Zahnzwischen-

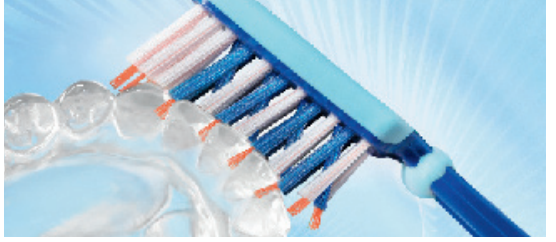

räume. „Ein hoher Druck gibt einzelnen Menschen vielleicht das Gefühl, gründlich geputzt zu haben, ist aber für ein gutes Reinigungsergebnis nicht notwendig und kann sogar das Zahnfleisch schädigen", sagt Dörfler. Daher gibt bei allen Dr.Best ${ }^{\oplus}$ Zahnbürsten die im Griff integrierte Flex-Zone bei zu hohem Druck nach.

$\boldsymbol{\lambda}$ Infos unter www.dr-best.de/produkte/ zahnbuerstenberater.jsp

\section{GC Germany}

\section{Niedrigste Schrumpfspannung durch neue Monomertechnologie}

Das Universalkomposit Kalore vereint drei Neuerungen in einem Hightechsystem: Dank des neu entwickelten Dentalmonomers DX-511 von DuPont, patentierten HDR-Füllern und einer verbesserten Füller-/Matrix-Verbindung lassen sich erstklassige Kompositrestaurationen von anhaltender Ästhetik und Funktionsfähigkeit verwirklichen. Kalore ist universell im Front- und Seitenzahn- bereich einsetzbar, ob für Klasse I- bis V-Kavitäten, bei keilförmigen Defekten oder bei Veneers. Mit seinem massiven Kern und den reaktionsstarken Seitenarmen ist das neue Monomer in Kalore vor allem für eine schrumpfarme Technologie verantwortlich. So bleibt die Adhäsion während und nach der Polymerisation zwischen den Mikrofüllern und der Matrix vollständig bestehen - ohne Verluste von Füller- oder Glaspartikeln. Dies führt zu einer weniger als zweiprozentigen Volumenschrump- fung und zu einer bislang nie erreichten minimierten Schrumpfspannung!

$\boldsymbol{\lambda}$ Tel.: 06172 - 99596-0 info@gcgermany.de www.gcgermany.de 